\title{
Rotating Models for Evolved Low-Mass Stars
}

\author{
Corinne Charbonnel \\ LA-OMP (CNRS UMR 5572) 14, av.Belin, F-31400 Toulouse and \\ Geneva Observatory, $\mathrm{CH}-1290$ Sauverny
}

Ana Palacios

LA-OMP (CNRS UMR 5572) 14, av.Belin, F-31400 Toulouse and IAA Université Libre de Bruxelles, Campus de la Plaine, Bd du Triomphe, B-1050 Bruxelles

\begin{abstract}
Low mass stars $\left(<2-2.5 \mathrm{M}_{\odot}\right)$ exhibit, at all the stages of their evolution, signatures of processes that require challenging modeling beyond the standard stellar theory. In this paper we focus on their peculiarities while they climb the red giant branch (RGB). We first compare the classical predictions for abundance variations due to the first dredge-up with observational data in various environments. We show how clear spectroscopic diagnostics probe the nucleosynthesis and the internal mixing mechanisms that drive RGB stars. Coherent data reveal in particular the existence of a non-standard mixing process that changes their surface abundances at the so-called RGB bump. By reviewing the models presented so far to explain the various abundance anomalies, we show that the occurrence of this extra-mixing process is certainly related to rotation. Finally we discuss the so-called Li-flash which is expected to occur at the very beginning of the extra-mixing episode.
\end{abstract}

\section{Abundance anomalies in RGB stars due to in situ processes}

\subsection{Observational data}

According to the classical stellar evolution theory ${ }^{1}$, the only opportunity for low mass stars (LMS) to modify their surface abundances happens on their way to the RGB when they do undergo the so-called first dredge-up (1DUP; Iben 1965). During this event their expanding convective envelope deepens in mass, leading to the dilution of the surface pristine material within regions that have undergone partial nuclear processing on the earlier main sequence. Qualitatively, this leads to the decrease of the surface abundances of the fragile $\mathrm{LiBeB}$ elements and of ${ }^{12} \mathrm{C}$, while those of ${ }^{3} \mathrm{He},{ }^{13} \mathrm{C}$ and ${ }^{14} \mathrm{~N}$ increase. Due to too low temperatures inside main sequence LMS, the abundances of $\mathrm{O}$ and heavier elements remain unchanged subsequent to the 1DUP. Quantitatively, these abundance variations depend on the stellar mass and metallicity (e.g. Sweigart, Greggio \& Renzini, 1989; Charbonnel 1994; Boothroyd \& Sackmann

\footnotetext{
${ }^{1}$ By this we refer to the modeling of non-rotating, non-magnetic stars, in which convection is the only mixing process considered.
} 
1999). After the 1DUP, the convective envelope withdraws while the hydrogen burning shell (HBS) moves outwards in mass. No more variations of the surface abundances are then expected until the star reaches the asymptotic giant branch.

The 1DUP predictions agree with the observations on the lower part of the $\mathrm{RGB}^{2}$. However observational evidences have accumulated, that we list below, of a second and distinct mixing episode that occurs in low mass stars after the end of the 1DUP, and more precisely at the RGB bump.

The determination of the carbon isotopic ratio $\left({ }^{12} \mathrm{C} /{ }^{13} \mathrm{C}\right.$, hereafter cir $)$ for RGB stars in open clusters with various turnoff masses (Gilroy 1989) provided the first pertinent clue on this process. It was indeed shown that bright RGB stars with initial masses lower than $\sim 2-2.5 \mathrm{M}_{\odot}$ exhibit cir considerably lower than predicted by the 1DUP. Thanks to data in stars sampling the RGB of M67 (Gilroy \& Brown 1991), it clearly appeared that observations deviated from classical predictions just at the so-called RGB bump (Charbonnel 1994). Field and globular cluster (GC) stars behave similarly ${ }^{3}$. In the former case this could be established thanks to the determination of the cir in large samples of stars for which Hipparcos parallaxes allowed the precise determination of their evolutionary status (Charbonnel, Brown \& Wallerstein 1998; Gratton et al. 2000). We knew for a long time that the brightest RGB stars in GCs presented cir close to the equilibrium value. But it is only this year that the region around the bump could be probed for two GCs : In NGC 6528 and M4, the cir drops below the 1DUP predictions just at the RGB bump (Shetrone 2002). The disagreement between observations and classical predictions is stronger for lower stellar mass and metallicity.

The extra-mixing process affects the surface abundances of other chemical elements : $\mathrm{Li}$ also decreases at the RGB bump, both in the field and in GCs (Pilachowski, Sneden \& Booth 1993; Grundahl et al. 2002). In field stars, C decreases while $\mathrm{N}$ increases for RGB stars brighter than the bump (Gratton et al. 2000) confirming the CN processing of the stellar envelope. The lowering of the $\mathrm{C}$ abundance along the RGB is also seen in GCs (e.g. Bellman et al. 2001 and references therein), though in this case the picture is more confused because of a probable non negligible dispersion of the initial $[\mathrm{C} / \mathrm{Fe}]$.

An in situ mechanism has also frequently been invoked to explain the abundance anomalies of heavier elements in GC stars, and in particular the O-Na anticorrelation. This pattern has been observed in the brightest GC RGB stars for a long time (see references in Ivans et al. 1999 and Ramirez \& Cohen 2002). However it is only thanks to $8-10 \mathrm{~m}$-class telescopes that $\mathrm{O}$ and $\mathrm{Na}$ abundances could be determined for less evolved stars, and in particular for turnoff stars in a couple of GCs (Gratton et al. 2001; Thévenin et al. 2001; Ramirez \& Cohen 2002). There, the O-Na anticorrelation extends to the main sequence. This result is crucial. Indeed, the $\mathrm{ON}$ - and $\mathrm{NeNa}$-cycles do not occur in main sequence

\footnotetext{
${ }^{2}$ One has of course to take into account possible variations of the surface Li abundance occurring already on the main sequence. This discussion is however out of the scope of the present paper (see Charbonnel, Deliyannis \& Pinsonneault 2000).
}

${ }^{3}$ The literature on the various abundance anomalies within GCs is so large that we chose to quote here only the most recent papers to illustrate our discussion. See Palacios (2002) for complete references. 
LMS, as the involved reactions require too high temperatures which are only reached when LMS are on the RGB. Thus the fact that the O-Na anticorrelation already exists on the main sequence clearly proves that it is not produced by in situ processes, but by external causes, the discussion of which is out of the scope of this paper (see Charbonnel 2002b).

Recently we have searched for an eventual evolution of the $\mathrm{O}$ and $\mathrm{Na}$ abundances with luminosity along the RGB for field stars. We gathered from the literature a large sample of field (625) stars with Hipparcos parallaxes over a large range of metallicity $([\mathrm{Fe} / \mathrm{H}]$ between -2.5 and 0$)$ for which we homogeneously redetermined the abundances of a large number of elements. No evolutionary effect could be found neither for $\mathrm{O}$ nor $\mathrm{Na}$ (Palacios et al. 2002a; Charbonnel et al. 2003), in agreement with the most recent GC data mentioned above.

Last but not least, the recent determination of the oxygen isotopic ratios in a couple of RGB stars with low cir (Balachandran \& Carr 2002) confirmed this result. In these objects indeed, both the ${ }^{16} \mathrm{O} /{ }^{17} \mathrm{O}$ and ${ }^{16} \mathrm{O} /{ }^{18} \mathrm{O}$ ratios appear to be high, in agreement with extensive $\mathrm{CN}$-processing but no dredge-up of ON-cycle material in RGB stars (see the arrow in Fig.1).

\subsection{Origin of the extra-mixing and its inhibition by $\mu$-gradients}

The observations summarized previously provide definitive clues on a second and distinct mixing episode that occurs in LMS when they reach the RGB bump. This process appears to be universal : it affects more than $95 \%$ of the LMS (Charbonnel \& do Nascimento 1998), whether they belong to the field, to open or globular clusters. Its signatures in terms of abundance anomalies are clear: the $\mathrm{Li}$ and the ${ }^{12} \mathrm{C}$ abundances as well as the ${ }^{12} \mathrm{C} /{ }^{13} \mathrm{C}$ ratio drop, while the ${ }^{14} \mathrm{~N}$ abundance and the ${ }^{16} \mathrm{O} /{ }^{18} \mathrm{O}$ ratio increase. On the other hand ${ }^{16} \mathrm{O},{ }^{17} \mathrm{O}, \mathrm{Na}$ and heavier elements remain unaffected. As we shall see in $\S 3$, this sequence may start with a very brief episode of $\mathrm{Li}$ enrichment.

These coherent data clearly indicate that during the extra-mixing episode at the RGB bump, the stellar convective envelope is connected with the radiative regions where CN-burning occurs, but not with the $\mathrm{ON}$ - and NeNa-processed layers (see Fig.1). As we shall discuss later on, the important increase of the molecular weight (or $\mu$ ) gradients when one approaches the HBS certainly shields these deeper regions from the extra-mixing.

$\mu$-gradients are also clearly responsible for the fact that the extra-mixing starts to be efficient only around the bump. Indeed during the 1DUP a $\mu$ discontinuity is built at the region of the deepest penetration of the convective envelope. This $\mu$-barrier then inhibits any mixing between the convective envelope and the HBS. At the RGB bump, the external regions of the HBS pass through the $\mu$-discontinuity. Due to the composition changes in the burning region, the stellar structure has to readjust, and the stellar luminosity then slightly decreases before increasing again. LMS stars spend a non negligible part of their RGB lifetime in this region; this explains why, for GCs for example, the bump clearly appears as a peak in the differential luminosity function or as a change in the slope of the cumulative luminosity function (e.g. Zoccali et al. 1999). After this evolutionary point, the $\mu$-gradients between the base of the convective envelope and the HBS are much smoother, allowing the occurrence of some 


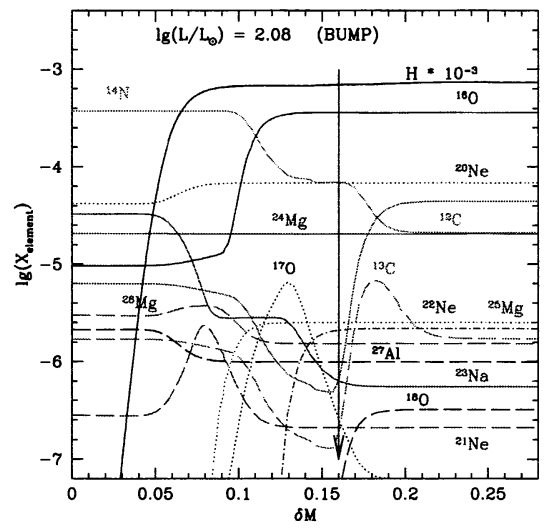

Figure 1. Abundance profiles of the isotopes participating to the $\mathrm{CNO}, \mathrm{NeNa}$ and $\mathrm{MgAl}$ cycles inside a $0.83 \mathrm{M}_{\odot},[\mathrm{Fe} / \mathrm{H}]=-1.6$ (typical of the GC M13) standard model at the luminosity of the RGB bump. The absissa is the relative mass coordinate $\delta M=\left(\mathrm{m}_{r}-\mathrm{m}_{B H B S}\right) /\left(\mathrm{m}_{B E C}-\mathrm{m}_{B H B S}\right)(\delta M=0$ at the bottom of the HBS, $\delta M=1$ at the base of the convective envelope). The vertical arrow indicates the maximum depth down to which the extra-mixing process is efficient according to the observations

extra-mixing in this radiative region (Sweigart \& Mengel 1979; Charbonnel 1994, 1995; Charbonnel et al. 1998).

All these observational facts represent strong clues on the origin of the extra-mixing process which is certainly rotationally-induced.

\section{Rotation-induced mixing and abundance anomalies in RGB stars}

\subsection{From meridional circulation ...}

Sweigart \& Mengel (1979, hereafter SM79) investigated the possibility that meridional circulation might lead to the mixing of CNO-processed material in RGB stars. Though the physics of the rotation-induced mixing invoked was incomplete, this pioneering work has magnificently settled the basis of a complex problem which is not definitively solved yet. ${ }^{4}$

SM79 discussed in great details the problem of $\mu$-gradients which were known to tend to inhibit meridional circulation (Mestel 1953, 1957). For the mixing to be efficient, they underlined the necessity for the radiative zone separating the convective envelope from the HBS not to have a significant gradient in $\mu$. As we said in $\S 1.2$, this condition is fulfilled only after the RGB bump, and, above this evolutionary point, only in the most external part of the HBS. This last point can be easily understood by looking at the abundance profiles inside a RGB star at the bump (Fig.1). When one moves inward the star from the base of the convective envelope $(\delta \mathrm{M}=1)$ toward the HBS $\left(\mathrm{X}_{\mathrm{H}}=0\right.$ at $\left.\delta \mathrm{M}=0\right)$,

\footnotetext{
${ }^{4}$ We do not discuss here the numerous parametric models that have been constructed to reproduce the abundance anomalies in RGB stars. We focus only on the physical models where the extra-mixing is assumed to be induced by rotation.
} 
one encounters first a shell in which $\mathrm{C}$ is burned into $\mathrm{N}$, then a C-depleted region and still deeper a shell in which $\mathrm{O}$ burns into $\mathrm{N}$. As underlined by SM79, the $\mathrm{H}$ abundance, and thus $\mu$, remain essentially constant across the $\mathrm{C}$ shell : the negligible $\mu$-gradients can not chocke the circulation currents in this region. However, the $\mu$-gradients become appreciable when one approaches the $\mathrm{O}$ shell due to the greater amount of $\mathrm{H}$ burning close to $\mathrm{ON}$ equilibrium. There, it is no longer clear whether the circulation currents can overcome the $\mu$-gradients, especially for the more metal-rich stars in which the $\mathrm{C}$ and $\mathrm{O}$ shells are shifted inward toward the HBS. Theoretical estimates of the $\mu$-gradients which are required to stop the meridional circulation were too uncertain for definitive conclusions to be put forward, though the metallicity dependance was soundly addressed.

The other crucial point made by SM79 concerned the importance of the angular momentum distribution within the deep convective envelope of the RGB star on the resulting CNO processing. Indeed, the angular velocity of a radiative layer near the HBS depends on how much angular momentum is left behind as the convective envelope retreats outward. SM79 investigated the two extreme cases, namely a constant specific angular momentum within the convective envelope, and a convective envelope rotating as a solid body. Substantial CNO processing of the envelope could be obtained with plausible main sequence angular velocity only if the inner part of the convective envelope was allowed to depart from solid body rotation. As we shall see in $\S 2.3$, our lack of knowledge of the distribution of angular momentum within the RGB convective envelope still remains nowadays one of the weakest points of the global picture.

\section{2. ... over a more complete description of rotation-induced mixing}

Rotational transport processes can not be simply reduced to meridional circulation. Once established indeed, this large scale circulation generates in turn advection of momentum and thus favours the development of various hydrodynamical instabilities ${ }^{5}$. Zahn (1992) proposed a description of the interaction between meridional circulation and shear turbulence, pushing forward the idea of chocking the meridional circulation by $\mu$-gradients. Following these developments, Charbonnel (1995, hereafter C95) re-investigated the influence of such a process in RGB stars. This framework is appealing because it takes advantage of some particularities of the non-homologous RGB evolution. In particular, some mixing is expected to take place wherever the rotation profile presents steep vertical gradients, as well as near nuclear burning shells. Moreover due to the stabilizing effect of the composition gradients, the mixing is expected to change the surface abundances on the RGB only after the bump.

Using a simplified version of Zahn's description, C95 showed that the rotationinduced mixing can indeed account for the observed behavior of carbon isotopic ratios and for the $\mathrm{Li}$ abundances in Population II low mass giants. Simultaneously, when this extra-mixing begins to act, ${ }^{3} \mathrm{He}$ is rapidly transported down to the regions where it burns by the ${ }^{3} \mathrm{He}(\alpha, \gamma){ }^{7} \mathrm{Be}$ reaction. This leads to a decrease of the surface value of ${ }^{3} \mathrm{He} / \mathrm{H}$ and of the final ${ }^{3} \mathrm{He}$ yields from LMS, solving the long-standing problem of the galactic evolution of ${ }^{3} \mathrm{He}$ (e.g. Charbonnel 2002a).

\footnotetext{
${ }^{5}$ We refer to the review by Suzanne Talon in these proceedings for a complete description of the transport processes inside rotating stars
} 
In these exploratory computations however, the transport of angular momentum by the hydrodynamical processes was not treated self-consistently, although it is of utmost importance in the understanding of the rotation-induced mixing.

\section{3. ... to a self-consistent treatment of the transport of angular momentum and of the chemicals}

Maeder \& Meynet (2000) nicely review the recent developments on the rotationinduced mixing in stars. They clearly state the fact that "the study of rotational mixing requires both an understanding of angular momentum evolution of stars and an understanding of the degree of mixing for a given angular momentum distribution. Angular momentum transport is the crucial ingredient for determining the extent of rotationally induced mixing". Let us now discuss the results obtained for RGB stars when the transport of angular momentum and of the chemicals as described by Zahn (1992), and latter on by Talon et al. (1997), Maeder \& Zahn (1998), and Palacios et al. (2003) is taken into account.

Two independant studies have been carried out using basically the same physical description of rotational mixing : Denissenkov \& Tout (2000, hereafter DT00) and Palacios et al. (2004, hereafter PCTF03). They differ on some details of the physical assumptions like the choice of the shear instability criteria and of the expression of the diffusion coefficient associated with vertical turbulence. These differences actually appear to be minor. Both studies obtain indeed very similar total diffusion coefficients for Pop II RGB stars at the bump, the vertical diffusion coefficient (turbulence) dominating over the effective diffusion coefficient (meridional circulation).

More importantly, however, a drastic assumption differentiates the work by DT00 from that of PCTF03 : DT00 indeed compute the transport of angular momentum and of the chemicals in a post-processing way; namely, they use the internal structure of a few standard RGB models to estimate the transport coefficients and the subsequent expected changes in the surface abundances. In this approach, there is no feedback of the mixing on the stellar structure and evolution which are computed independently in the standard way.

On the other hand, PCTF03 do treat the transport of both angular momentum and of the chemicals inside their stellar evolution code, thereby following the "non-standard" evolution of the star from the zero age main sequence on. As a result in this case, the mixing has a feedback on the star since its early evolution. On the RGB in particular, the mixing distorts the stationary profiles of the chemicals deep inside the star even if the surface abundances are not yet affected; it alters for example the positions of the $\mathrm{C}$ and $\mathrm{O}$ depleted regions. In turn, the mixed star is allowed to react to the mixing that it undergoes, thereby influencing the mixing itself.

This difference has a strong influence on the final predictions. In DT00 indeed, the transport is applied on standard profiles, and surface variations of $\mathrm{O}$ and $\mathrm{Na}$ are expected in RGB stars. In PCTF03 however, the mixing is applied on self-consistently distorted profiles; as a result, only variations of the $\mathrm{C}$ isotopes and of $\mathrm{N}$ are predicted at the surface of the star. These latter predictions are in better agreement with the observations described in $\S 1$. 


\subsection{Clues from horizontal branch stars}

Let us note an important point. In all these studies (SM79, DT00, PCTF03), notable changes of the surface abundances could be reached only under the assumption of constant specific angular momentum within the convective envelope (see §2.1). Indeed in the case of solid body rotation prohibitively high values of the main sequence surface rotation velocities are required to produce enough mixing. The same result has been obtained by Chanamé, Pinsonneault \& Terndrup (these proceedings) in their simplified rotating Pop I RGB model.

Unfortunately, the distribution of angular momentum within the very deep convective envelope of an RGB star is far from being understood. More than 20 years after SM79, one is still reduced to use the abundance anomalies on the RGB as evidences for some departure from solid-body rotation (see also Kumar, Narayan \& Loeb 1995). This assumption is actually sustained by the study of horizontal branch stars which provides some intriguing clues about angular momentum evolution on the RGB.

Peterson (1983) first discovered that some blue horizontal branch stars are rapid rotators. Pinsonneault et al. (1991) noted that the combination of RGB mass loss, high horizontal branch rotation rate, and low main sequence rotation required strong differential rotation with depth in giants. If the convection zone of RGB stars had solid body rotation, differential rotation with depth in their MS precursors was also required. Behr et al. (2000) found a break in the rotational properties of horizontal branch stars, in the sense that very blue horizontal branch stars both exhibited the surface signature of atomic diffusion and rotated more slowly than slightly cooler stars. Sills \& Pinsonneault (2000) interpreted this as an evidence that mean molecular weight gradients caused by atomic diffusion inhibit angular momentum transport in hot horizontal branch stars. This is an independent test of the impact of composition gradients in a different evolutionary phase (for discussions of the effects on the main sequence see for example Vauclair 1999; Théado \& Vauclair 2001; Palacios et al. 2003).

In addition, Sills \& Pinsonneault (2000) found that uniform rotation at the main sequence turnoff was only compatible with rapid horizontal branch rotation under the following conditions: (1) turnoff rotation of order $4 \mathrm{~km} / \mathrm{s}$ rather than the $1 \mathrm{~km} / \mathrm{s}$ inferred from an extrapolation of the Population I angular momentum loss law to Population II stars; (2) constant specific angular momentum in the convective envelopes of giants; (3) strong differential rotation with depth in the radiative cores of giants.

All of these are different from the expectations from main sequence angular momentum evolution models, and they are an indication that further theoretical work is needed in physical models of RGB rotational mixing. It is encouraging, however, that all of the above properties favor more vigorous rotational mixing on the RGB than would be expected from the opposite conclusions.

\section{The Li flash}

A few $(\sim 1 \%)$ RGB stars present Li-overabundances (see also Drake et al. and Konstantinova-Antova in these proceedings). The fact that these so-called super Li-rich giants are all located at the RGB bump lead Charbonnel \& Balachandran (2000, hereafter CB00) to conclude that the Li-rich phase was a precursor to the 
extra-mixing process described previously. This brought very tight constraints on the underlying physics.

Denissenkov \& Weiss (2000, hereafter DW00) investigated the effect of rotation-induced mixing on the surface lithium abundance using the transport coefficients $\mathrm{D}_{\mathrm{R}}$ derived by DT00 (see $\S 2.3$ ). They found that the corresponding values of $D_{R}\left(10^{8}\right.$ to $\left.10^{10} \mathrm{~cm}^{2} \mathrm{sec}^{-1}\right)$ were too low to lead to lithium enrichment and invoked the engulfing of a planet in order to trigger and dope the transport process. While this combined scenario is rather attractive, it is harmed by the fact that it can happen at any time on the RGB while the super-Li rich giants with solar metallicity discovered up to now do stand by the bump (CB00). However what was not investigated by DT00 and DW00 was the structural response of the star to the extra-mixing. Indeed, as already discussed in $\S 2.3$, their computations of the transport coefficients and of the abundance variations were done in a post-processing approach where standard stellar structures were used as background models.

Palacios, Charbonnel \& Forestini (2001) proposed that the structural response to the mixing could actually be the cause of the increase of $D_{R}$ which is necessary to produce super-Li rich giants. The mixing sequence is the following. At the RGB bump the external wing of the ${ }^{7} \mathrm{Be}$ peak crosses the molecular weight discontinuity before the ${ }^{13} \mathrm{C}$ peak does, and is then connected with the convective envelope by the extra-mixing process. ${ }^{7} \mathrm{Be}$ produced via ${ }^{3} \mathrm{He}(\alpha, \gamma){ }^{7} \mathrm{Be}$ starts to diffuse outwards. However, due to the relatively low $\mathrm{D}_{\mathrm{R}}$ given above, the transported ${ }^{7} \mathrm{Be}$ decays in regions where ${ }^{7} \mathrm{Li}$ is rapidly destroyed by proton capture. A lithium burning shell appears. ${ }^{7} \operatorname{Li}(\mathrm{p}, \alpha) \alpha$ becomes the dominant reaction leading to an increase of the local temperature and of both the local and total luminosities. Palacios et al. suggested that the meridional circulation and the corresponding transport coefficient then increase due to the $\epsilon_{\text {nuc }}\left({ }^{7} \mathrm{Li}+\mathrm{p}\right)$ burst, allowing for lithium enrichment of the convective envelope. A lithium flash terminates this phase ${ }^{6}$. As the convective instability develops around the Li-burning shell, it erases the molecular weight gradient and the mixing is free to proceed deeper. Both the lithium abundance and the carbon isotopic ratio then drop at the stellar surface. When the flash is quenched, the external convective envelope deepens again. We suggest that this creates a new $\mu$-barrier, similar to that built up during the 1DUP, which then inhibits again the extra-mixing during the subsequent evolution.

During the whole sequence described above the temporary increase of the stellar luminosity causes an enhanced mass loss rate which naturally accounts for the dust shell suggested by the far-infrared color excesses measured for some lithium-rich objects from IRAS fluxes (de la Reza et al. 1996, 1997). Let us note that the contribution of these stars to the lithium enrichment of the Galaxy should be very modest.

\footnotetext{
${ }^{6}$ Our scenario is different from the previous works which explored the possible occurrence of shell flashes on the RGB and which considered only the reactions of the CNO cycle (Bolton \& Eggleton 1973; Dearborn et al. 1975; Von Rudloff et al. 1988). It also differs from the model developed by Fujimoto et al. (1999) to explain $\mathrm{Mg}$ and $\mathrm{Al}$ anomalies in globular cluster red giants. These authors indeed proposed that a flash is triggered off deeper in the HBS due to the inward mixing of $\mathrm{H}$ down into the degenerate $\mathrm{He}$ core.
} 


\section{Conclusions}

During the last three decades, an incredible amount of work has been devoted to the understanding of the chemical anomalies exhibited by evolved LMS. On the observational side, crucial breakthroughs were made possible recently thanks to the advent of 8-10m-class telescopes. The signatures of the extra-mixing process that occurs at the RGB bump are now very clear.

On the other hand, recent theoretical models include a sophisticated description of the mixing processes induced by rotation. Those which consistently couple the transport of angular momentum and of the chemicals with the structural evolution of the star predict surface abundance variations on the RGB in agreement with the observational data. However, a couple of assumptions remain to be tested. One of the main uncertainties concerns the distribution of angular momentum within the deep convective envelope. A final answer to this problem certainly necessitates $3 \mathrm{D}$ hydrodynamical simulations. Another important point which remains to be investigated is the reaction of the meridional circulation and of the various instabilities to a major and local release of nuclear energy.

Rotation-induced mixing processes modify all the stellar outputs, and in particular the final yields from LMS (i.e., ${ }^{3} \mathrm{He}$ is not over-produced anymore by rotating LMS). Their impacts on the stellar lifetimes and on the further evolution (luminosity of the RGB tip, morphology of the horizontal branch, AGB phase, ...) have to be investigated now in a systematic way. This is of crucial importance if one wants to correctly understand the role of LMS in the evolution (chemical and spectro-photometric) of stellar clusters and of galaxies in general.

Acknowledgments. We thank the french Programme National de Physique Stellaire and Programme National Galaxies for their support on this work. AP aknowledges financial support from the IAU.

\section{References}

Balachandran, S., Carr, J. 2002, in: C.Charbonnel, D.Schaerer, \& G.Meynet (eds.), CNO in the Universe, ASP Conference Series, p. 101

Behr, B.B., Cohen, J.G., McCarthy, J.K. 2000, ApJ 531, L37

Bellman, S., et al., 2001, PASP 113, 326

Bolton, A.J.C., Eggleton, P.P., 1973, A\&A 24, 429

Boothroyd, A.I., Sackmann, I.J., 1999, ApJ 510, 232

Charbonnel, C., 1994, A\&A 282, 811

Charbonnel, C., 1995, ApJ 543, L41

Charbonnel, C., 2002a, Nature 415, 27

Charbonnel, C., 2002b, in: C.Charbonnel, D.Schaerer, \& G.Meynet (eds.), CNO in the Universe, ASP Conference Series, in press

Charbonnel, C., Balachandran, S., 2000, A\&A 359, 563, CB00

Charbonnel, C., Brown, J.A., Wallerstein, G., 1998, A\&A 332, 204

Charbonnel, C., do Nascimento, J., 1998, A\&A 336, 915 
Charbonnel, C., Deliyannis, C., Pinsonneault, M., 2000, in: Da Silva et al. (eds), The Light Elements and their Evolution, Proc. IAU Symp. 198, 87

Charbonnel, C., Palacios, A., Thévenin, F., Bolmont, J., 2004, in preparation

Dearborn, D.D., Bolton, A.J.C., Eggleton, P.P., 1975, MNRAS 170, 7

de la Reza, R., Drake, N.A., da Silva, L., 1996, ApJ 456, L115

de la Reza, R., Drake, N.A., da Silva, L., Torres, C.A.O., Martin, E.L., 1997, ApJ 482, L77

Denissenkov, P.A., Tout, C.A., 2000, MNRAS 316, 395

Denissenkov, P.A., Weiss, A., 2000, A\&A 358, L49, DW00

Fujimoto, M.Y., Aikawa, M., Kato, K., 1999, ApJ 519, 733

Gilroy, K.K., 1989, ApJ 347, 835

Gilroy, K.K., Brown, J., 1991, ApJ 371, 578

Gratton, R.G., Bonifacio, P., Bragaglia, A., et al., 2001, A\&A 369, 87

Gratton, R.G., Sneden, C., Carreta, E., Bragaglia, A., 2000, A\&A 354, 169

Grundahl, F., Briley, M., Nissen, P.E., Feltzing, S., 2002, A\&A 336, 915

Iben, I. Jr, 1965, ApJ 142, 1447

Ivans, I.I., Sneden, C., Kraft, R.P., et al., 1999, AJ 118, 1273

Kumar, P., Narayan, R., Loeb, A., 1995, ApJ 453, 480

Maeder, A., Meynet, G., 2000, ARA\&A38, 143

Maeder, A., Zahn, J.P., 1998, A\&A 334, 1000

Mestel, L., 1953, MNRAS 113, 716

Mestel, L., 1957, ApJ 126, 550

Palacios, A., 2002, PhD thesis, UPS Toulouse III

Palacios, A., Charbonnel, C., Bolmont, J., Thévenin, F., 2002a, Ap\&SS 281, 213

Palacios, A., Charbonnel, C., Forestini, M., 2001, A\&A 375, L9

Palacios, A., Talon, S., Charbonnel,C., Forestini, M., 2003, A\&A 399, 603

Palacios, A., Charbonnel, C., Talon, S., Forestini, M., 2004, A\&A in preparation

Peterson, R.C., 1983, ApJ 275, 737

Pilachowski, C.A., Sneden, C., Booth, J., 1993, ApJ 407, 699

Pinsonneault, M.H., Deliyannis, C.P., Demarque, P., 1991, ApJ 367, 239

Ramirez, S.V., Cohen, J.G., 2002, AJ 123, 3277

Sills, A., Pinsonneault, M.H., 2000, ApJ 544, 944

Shetrone, M., 2002, in: C.Charbonnel, D.Schaerer, \& G.Meynet (eds.), CNO in the Universe, ASP Conference Series, in press

Sweigart, A.V., Greggio, L., Renzini, A., 1989, ApJ 69, 911

Sweigart, A.V., Mengel, J.G. 1979, ApJ 229, 624, SM79

Talon, S., Zahn, J.P., Maeder, A., Meynet, G., 1997, A\&A 322, 209

Thévenin, F., Charbonnel, C., de Freitas Pacheco, J.A., et al., 2001, A\&A 373, 905

Vauclair, S., 1999, A\&A 351, 973

Vauclair, S., Théado, S., 2001, A\&A 375, 70

Von Rudloff, I.R., VandenBerg, D.A., Hartwick, F.D.A., 1988, ApJ 324, 840

Zahn, J.P., 1992, A\&A 265, 115

Zoccali, M., Cassisi, S., Piotto, G., Bono, G., Salaris, M., 1999, ApJ 518, L49 